\title{
Female-specific risk of Alzheimer's disease is associated with tau phosphorylation processes: A transcriptome-wide interaction analysis.
}

\author{
Alejandro Cáceres ${ }^{1,2, *}$ and Juan R. González ${ }^{1,2,3}$ \\ ${ }^{1}$ Instituto de Salud Global de Barcelona (ISGlobal), Barcelona 08003, Spain \\ ${ }^{2}$ Centro de Investigación Biomédica en Red en Epidemiología y Salud Pública (CIBERESP), \\ Spain \\ ${ }^{3}$ Department of Mathematics, Universitat Autònoma de Barcelona, Bellaterra (Barcelona) \\ 08193, Spain
}

*Correspondence to Alejandro Cáceres: alejandro.caceres@isglobal.org

\section{Key Words}

Alzheimer's disease, female risk, sexual dimorphism, gene expression, transcriptome, expression array, transcriptome, tau, phosphorylation, neurofibrillary tangles, NCL, KIF2A, transcriptome-wide interaction analysis, differential expression.

\begin{abstract}
The levels of tau phosphorylation differ between sexes in Alzheimer's disease (AD). Transcriptome-wide associations of sex by disease interaction could indicate whether specific genes underlie sex differences in tau pathology, however, no such study has been reported yet. We report the first analysis of the effect of the interaction between disease status and sex on differential gene expression, meta-analyzing transcriptomic data from the three largest publicly available case-control studies $(\mathrm{N}=776)$ in brain to date. 121 genes, significantly associated with sex-AD interactions, were enriched in phosphoproteins (FDR=9.65 $\times 10^{-6}$ ). High and consistent associations were found for the over-expressions of NCL (FDR=0.0020), whose phosphorylated protein generates an epitope against neurofibrillary tangles, and KIF2A (FDR=0.01), a microtubule-associated motor protein gene. Transcriptome-wide interaction analyses suggest sex-modulated tau phosphorylation, at sites like Thr231, Ser199 or Ser202 that could increase the risk of women to $A D$ and indicate sex-specific strategies for intervention and prevention.
\end{abstract}




\section{Introduction}

Two-thirds of Alzheimer's disease patients are women (Association et al., 2018). One contributor to the higher prevalence of $A D$ in women is survival bias, as men at risk are more likely to die before developing $A D$ than women at risk (Mielke et al., 2014). However, sexbiased, environmental or biological risk factors also contribute to the difference in $A D$ risk, such as educational attainment or the APOE-e4 genotype among others (Stern et al., 1994; Altmann et al., 2014; Ferretti et al., 2018). Evidence indicates, in particular, important differences in tau cerebrospinal fluid in women with amyloidosis (Hohman et al., 2018), supported by recently observed differences in the production and the structure of neurofibrillary tangles between sexes (Buckley et al., 2019). Depletion of hormones, in particular in estrogen, a known risk factor of $A D$ (Merlo et al., 2017), could play important roles in female tauopathy as hormones can influence tau phosphorylation at specific sites (Muñoz-Mayorga et al., 2018).

While genetic studies have shown that tau pathology is higher in female APOE-e4 carriers (Altmann et al., 2014), it is unknown which other genes could be linked to sex-specifictauopathy processes. Recent studies have found sex, and $A D$ by sex differences in gene expression in the brain in a few individuals (Shi et al., 2016; Mathys et al., 2019). However, there is no large study that identifies transcriptome-wide signatures of sex-AD interactions. To fill this gap, we performed a meta-analysis of transcriptomic data from the three largest, publicly available, transcriptomic studies to date. We aimed to find which genes showed a differential expression that was specific to female $A D$ patients, and contrast the findings with relevant sex-biased risk factors of $A D$, such as processes involved in tau pathology.

\section{Methods}

\subsection{Statistical Analyses}

We performed transcriptomic-wide interaction analysis on large AD case-control studies. For each study, we tested the association between gene expression and the interaction between sex and AD status using the linear model 


$$
E_{i j}=\alpha_{i}+\beta_{i}\left(S_{j} \times D_{j}\right)+\sum_{r=1 \ldots k} Y_{i r j} C_{r j}+\varepsilon_{i j}
$$

where Eij is the gene expression at probe $\mathrm{i}$ for individual $\mathrm{j}$ with sex $\mathrm{Sj}$ and disease status $\mathrm{Dj}$. $\mathrm{Crj}$ are $\mathrm{k}$ covariates that include sex, disease status, age, post-mortem interval and batch effects. Coding sex as Female: 1 and Male: 0, and Alzheimer status as AD: 1 and control: 0, i is the specific effect of AD-females on the transcription of probe i. For each common gene across studies, we meta-analyzed the associations with the sex-AD interaction, using an adaptive weighted combination of P-values, as implemented in the Bicoductor's library MetaDE. We adjusted the one-tailed testing of the combination by combining the P-values for each tail across the studies and selected the direction of the test with the most significant association. P-values were then corrected for multiple comparisons with false discovery rate (FDR). We also performed enrichment pathway analyses for the significant genes.

\subsection{Expression Data}

We identified three large case-control transcriptomic studies $(N>70)$ of $A D$ in the GEO repository. These have accession numbers GSE15222, GSE44770 and GSE84422 (see Table 1). The three studies collected microarray transcriptomic data from cortical samples including frontal, temporal and visual cortices. The study GSE15222 contains transcriptomic data in cortical tissue of non-demented individuals and late-onset Alzheimer's disease (LOAD) patients. GSE44770/GSE44771 is a case/control transcriptomic study of LOAD based on autopsied tissues from the dorsolateral prefrontal cortex (DLPF) and visual cortex (VC). In GSE84422, 19 brain regions from 125 brains were used for transcriptomic analysis, with an average of 50 to 60 subjects per brain region. We selected individuals sampled in only one cortex among DPFC, PFC and VC. For individuals with multiple samples in the cortices, data for one cortex was randomly selected, such that only one individual had data for only one cortex. 
The numbers of females and $A D$ cases varied across studies, where GSE84422 had the largest ratios of $A D$-females. Overall, the studies comprised a total of 858 individuals, 384 (44.7\%) females and 473 (55.1\%) AD cases.

\subsection{Software}

We used Bioconductor and $\mathrm{R}$ packages to download and analyze the transcriptomic data of all four studies using a common pipeline. Quality controlled and normalized transcriptomic and phenotypic data were downloaded with the GEOquery package. Variables that represented underlying technical (batch) effects were estimated using surrogate variable analyses (SVA package), Differential expression analyses for the sex-AD interaction was performed using the limma package. Models were adjusted by sex, $A D$ status, age, post mortem interval and the surrogate variables for each study. Meta-analyses were performed with the MetaDE package, and enrichment analyses with STRING using the Uniprot database. The entire reproducible code and analyses can be found in the supplementary material.

\section{Results}

\subsection{Transcriptome-wide meta-analysis in cerebral cortex}

We first analyzed the sex-specific transcriptome-wide signatures of $A D$ in each study, testing the association between gene expression and the sex-AD interaction. P-values for the differential expression of 10,795 common genes were combined across the three studies and adjusted for multiple comparisons. We discarded genes in chromosome $\mathrm{Y}$. We observed 70 statistically significant genes after FDR correction (Table S1-S2). Many of these genes have been previously linked with tau phosphorylation and AD.

The top three genes that significantly associated with the sex-AD interaction, from the metaanalysis (Tables S1-S2) were NCL (FDR $=0.0022)$, TARDBP $(F D R=0.0030)$, and KIF2A (FDR $=0.0052)$. In particular, NCL and KIF2A showed consistent nominal association in two different studies, where both up-regulated in $A D$ females with respect to female controls but down-regulated for $A D$ males with respect to male controls (Figure $1 A$ ). These genes are 
linked to tau phosphorylation. NCL encodes the nucleolin protein, whoe phosphorylation by Cdc2 kinase generates the TG-3 epitope against neurofibrillary tangles (Dranovsky et al., 2001). KIF2A encodes the microtubule-associated motor protein gene that is phosphorylated by tau tubulin kinase 2 (TTBK2) (Sato et al., 2006). TARDBP is also targeted by TTBK2 in frontotemporal lobar degeneration (Taylor et al., 2018), and its rare p.Ala90Val substitutions in exon 3 were associated to AD (Brouwers et al., 2010). Top associated genes also included DNAJB6 (FDR=0.0098), a chaperone molecule that inhibits amyloid-beta concentrations (Månsson et al., 2014), and PPP2R2A (FDR = 0.01), involved in site-specific regulation of tau phosphorylation (Yu et al., 2014). In summary, we observed numerous genes whose expression levels associated with the sex-AD interaction, many of which with direct links to $A D$ through tau-phosphorylation processes.

\subsection{Enrichment meta-analysis}

We performed a gene enrichment analysis for the 128 genes that significantly deregulated in AD-females, to identify the molecular mechanisms that could underlie these associations (Table S3). We observed that the 128 genes were highly connected in a protein-protein interaction network $\left(P=1.6 \times 10^{-5}\right)$ (Figure $\left.1 B\right)$. The network was strongly enriched with phosphoproteins (FDR =0.0016) and neuropathy (FDR=0.026), in line with the single gene associations we had found. We also observed significant enrichment with acetylation $\left(\mathrm{FDR}=2.05 \times 10^{-5}\right)$, Ubl conjugation $\left(\mathrm{FDR}=3.4 \times 10^{-4}\right)$, isopeptide bonds $(\mathrm{FDR}=0.0012)$, mitochondrion (FDR=0.026) and nucleotide-binding (FDR=0.035), Figure 1C.We did not observe any significantly enriched pathway from Kyoto encyclopedia of genes and genomes (KEGG). We also performed a sensitivity analysis, removing the samples from the visual cortex; less affected by $\mathrm{AD}$, and observed consistent results in genes and pathways (Tables S4-S5).

\section{Discussion}

Compelling evidence indicates that the presence and fate of neurofibrillary tangles by tau hyperphosphorylation in $A D$ is different between men and women (Buckley et al., 2019). Differences in sex hormones could explain these differences (Muñoz-Mayorga et al., 2018). In 
particular, menopause and oophorectomy could increase AD-risk by the removal protection by estrogen and progesterone against tau phosphorylation by GSK3 (Merlo et al., 2017). In this context, our analyses indicate specific processes and phosphorylation sites that could be involved in the sex differences in AD tauopathy. For instance, the epitope TG-3, generated by the phosphorylation of nucleolin (Dranovsky et al., 2001), is specific of tau phosphorylation at Thr231. Interestingly, pThr231 tau is found to be attenuated by 17betaestradiol (E2) (Shi et al., 2008) and to increase in the latter stages of AD (Braak stage V/VI) (Neddens et al., 2018), suggesting a sex-specific evolution of tau phosphorylation mediated by estrogen at Thr231 and giving mechanistic support to our observation of NCL over-expression in $A D$ females. Notably, E2 also attenuates tau phosphorylation at Ser199, Ser202, targets of TTBK1 in addition to Ser135 of KIF2A (Sato et al., 2006), which can also be linked to neurofibrillary tangles by its microtubule functions (Homma et al., 2003), and that we also observed consistently overexpressed in AD females. While we remarkably also observed associations with PPP2R2A, which appears to precisely reduce tau phosphorylation at Ser199, Ser202/Thr205 and Thr231 (Yu et al., 2014), we noted that its over-expression unexpectedly correlated with $\mathrm{AD}$ females. It is important to note, however, that our results are transcriptome-wide hypothesis-free findings and, therefore, they require further validation of the concrete observations they reveal, together with their interrelation. Our results suggest that specific phosphorylation sites of tau should be further investigated for precision treatment and risk assessment of women. More generally, our results importantly provided consistent and numerous evidence on the sex differences in tau phosphorylation in $A D$ etiology. $A D$ is among several diseases that show sexual dimorphism in risk and progression. Deepening into the biological causes of these differences will help to design sexspecific treatments and prevention. We showed that, in the case of $A D$, interaction analyses between sex and phenotypes can provide relevant transcriptomic signatures of sexual dimorphism. Consequently, the analyses could also be informative in other sexually dimorphic traits and diseases.

\section{Conflicts of interest:}

All authors declare no conflict of interests.

\section{Funding}


This research has received funding from the Spanish Ministry of Education, Innovation and Universities, the National Agency for Research and the Fund for Regional Development (RTI2018-100789-B-I00) and the Catalan Department of Economy and Knowledge (SGR2017/801).

\section{Acknowledgements}

We would like to thank the reviewer for insightful comments.

\section{References}

Altmann, A., Tian, L., Henderson, V.W., Greicius, M.D., Investigators, A.D.N.I., 2014. Sex modifies the apoe-related risk of developing Alzheimer disease. Annals of neurology $75,563-$ 573.

Association, A., et al., 2018. 2018 alzheimer's disease facts and figures. Alzheimer's \& Dementia 14, 367-429.

Brouwers, N., Bettens, K., Gijselinck, I., Engelborghs, S., Pickut, B.A., Van Miegroet, H., Montoya, A.G., Mattheijssens, M., Peeters, K., De Deyn, P.P., et al., 2010. Contribution of tardbp to alzheimer's disease genetic etiology. Journal of Alzheimer's Disease 21, 423-430.

Buckley, R.F., Mormino, E.C., Rabin, J.S., Hohman, T.J., Landau, S., Hanseeuw, B.J., Jacobs, H.I., Papp, K.V., Amariglio, R.E., Properzi, M.J., et al., 2019. Sex differences in the association of global amyloid and regional tau deposition measured by positron emission tomography in clinically normal older adults. JAMA neurology 76, 542-551.

Dranovsky, A., Vincent, I., Gregori, L., Schwarzman, A., Colflesh, D., Enghild, J., Strittmatter, W., Davies, P., Goldgaber, D., 2001. Cdc2 phosphorylation of nucleolin demarcates mitotic stages and alzheimer's disease pathology. Neurobiology of aging 22, 517-528. 
Ferretti, M.T., lulita, M.F., Cavedo, E., Chiesa, P.A., Dimech, A.S., Chadha, A.S., Baracchi, F., Girouard, H., Misoch, S., Giacobini, E., et al., 2018. Sex differences in alzheimer diseaseâ $\square \square$ the gateway to precision medicine. Nature Reviews Neurology , 1.

Hohman, T.J., Dumitrescu, L., Barnes, L.L., Thambisetty, M., Beecham, G., Kunkle, B., Gifford, K.A., Bush, W.S., Chibnik, L.B., Mukherjee, S., et al., 2018. Sex-specific association of apolipoprotein e with cerebrospinal fluid levels of tau. JAMA neurology 75, 989-998. Homma, N., Takei, Y., Tanaka, Y., Nakata, T., Terada, S., Kikkawa, M., Noda, Y., Hirokawa, N., 2003. Kinesin superfamily protein 2 a (kif2a) functions in suppression of collateral branch extension. Cell 114, 229-239.

Månsson, C., Arosio, P., Hussein, R., Kampinga, H.H., Hashem, R.M., Boelens, W.C., Dobson, C.M., Knowles, T.P., Linse, S., Emanuelsson, C. 2014. Interaction of the molecular chaperone dnajb6 with growing amyloid-beta 42 (a 42) aggregates leads to sub-stoichiometric inhibition of amyloid formation. Journal of Biological Chemistry 289, 31066-31076.

Mathys, H., Davila-Velderrain, J., Peng, Z., Gao, F., Mohammadi, S., Young, J.Z., Menon, M., He, L., Abdurrob, F., Jiang, X., et al., 2019. Single-cell transcriptomic analysis of alzheimer's disease. Nature , 1.

Merlo, S., Spampinato, S.F., Sortino, M.A., 2017. Estrogen and alzheimer's disease: Still an attractive topic despite disappointment from early clinical results. European journal of pharmacology 817, 51-58.

Mielke, M.M., Vemuri, P., Rocca, W.A., 2014. Clinical epidemiology of alzheimer's disease: assessing sex and gender differences. Clinical epidemiology 6, 37.

Muñoz-Mayorga, D., Guerra-Araiza, C., Torner, L., Morales, T., 2018. Tau phosphorylation in female neurodegeneration: role of estrogens, progesterone, and prolactin. Frontiers in Endocrinology 9, 133. 
Neddens, J., Temmel, M., Flunkert, S., Kerschbaumer, B., Hoeller, C., Loeffler, T., Niederkofler, V., Daum, G., Attems, J., Hutter-Paier, B., 2018. Phosphorylation of different tau sites during progression of alzheimer's disease. Acta neuropathologica communications 6, 52. Sato, S., Cerny, R.L., Buescher, J.L., Ikezu, T., 2006. Tau-tubulin kinase 1 (ttbk1), a neuronspecific tau kinase candidate, is involved in tau phosphorylation and aggregation. Journal of neurochemistry 98, 1573-1584.

Shi, H.R., Zhu, L.Q.,Wang, S.H., Liu, X.A., Tian, Q., Zhang, Q.,Wang, Q.,Wang, J.Z., 2008. 17-estradiol attenuates glycogen synthase kinase-3 activation and tau hyperphosphorylation in akt-independent manner. Journal of neural transmission 115, 879-888.

Shi, L., Zhang, Z., Su, B., 2016. Sex biased gene expression profiling of human brains at major developmental stages. Scientific reports 6, 21181.

Stern, Y., Gurland, B., Tatemichi, T.K., Tang, M.X., Wilder, D., Mayeux, R., 1994. Influence of education and occupation on the incidence of alzheimer's disease. Jama 271, 1004-1010.

Taylor, L.M., McMillan, P.J., Liachko, N.F., Strovas, T.J., Ghetti, B., Bird, T.D., Keene, C.D., Kraemer, B.C., 2018. Pathological phosphorylation of tau and tdp-43 by ttbk1 and ttbk2 drives neurodegeneration. Molecular neurodegeneration 13, 7.

Yu, U.Y., Yoo, B.C., Ahn, J.H., 2014. Regulatory b subunits of protein phosphatase 2a are involved in site-specific regulation of tau protein phosphorylation. The Korean Journal of Physiology \& Pharmacology 18, 155-161. 


\section{Table and Figure Legends}

\begin{tabular}{|l|c|c|c|c|c|c|c|c|}
\hline Study & Cortical region & $\mathbf{N}$ & $\begin{array}{c}\text { Males } \\
\text { (AD/control) }\end{array}$ & $\begin{array}{c}\text { Females } \\
\text { (AD/control) }\end{array}$ & Mean age & $\begin{array}{c}\text { Mapped } \\
\text { genes }\end{array}$ & Platform \\
\hline GSE15222 & $\begin{array}{c}\text { Frontal/ } \\
\text { Temporal/unreported }\end{array}$ & 311 & $\begin{array}{c}157 \\
(62 / 95)\end{array}$ & $\begin{array}{c}154 \\
(70 / 84)\end{array}$ & 82.3 & 15612 & Sentrix HumanRef-8 & 19361613 \\
\hline $\begin{array}{l}\text { GSE44770/ } \\
\text { GSE44771 }\end{array}$ & $\begin{array}{c}\text { Dorsolateral prefrontal/ } \\
\text { Visual }\end{array}$ & 390 & $\begin{array}{c}255 \\
(110 / 1145)\end{array}$ & $\begin{array}{c}135 \\
(104 / 31)\end{array}$ & 71.5 & 18121 & Rosetta/Merck 44k 1.1 & 23622250 \\
\hline GSE84422 & $\begin{array}{c}\text { Dorsolateral Prefrontal/ } \\
\text { Prefrontal/Visual }\end{array}$ & 84 & $\begin{array}{c}25 \\
(8 / 17)\end{array}$ & $\begin{array}{c}59 \\
(10 / 49)\end{array}$ & 85.7 & 13516 & Affymetrix U133 Plus 2.0 & 27799057 \\
\hline
\end{tabular}

Table 1. Large case-control transcriptomic studies of AD identified in the GEO repository. The three studies in post mortem brain tissue were meta-analyzed. In all studies cortical regions were assayed. 

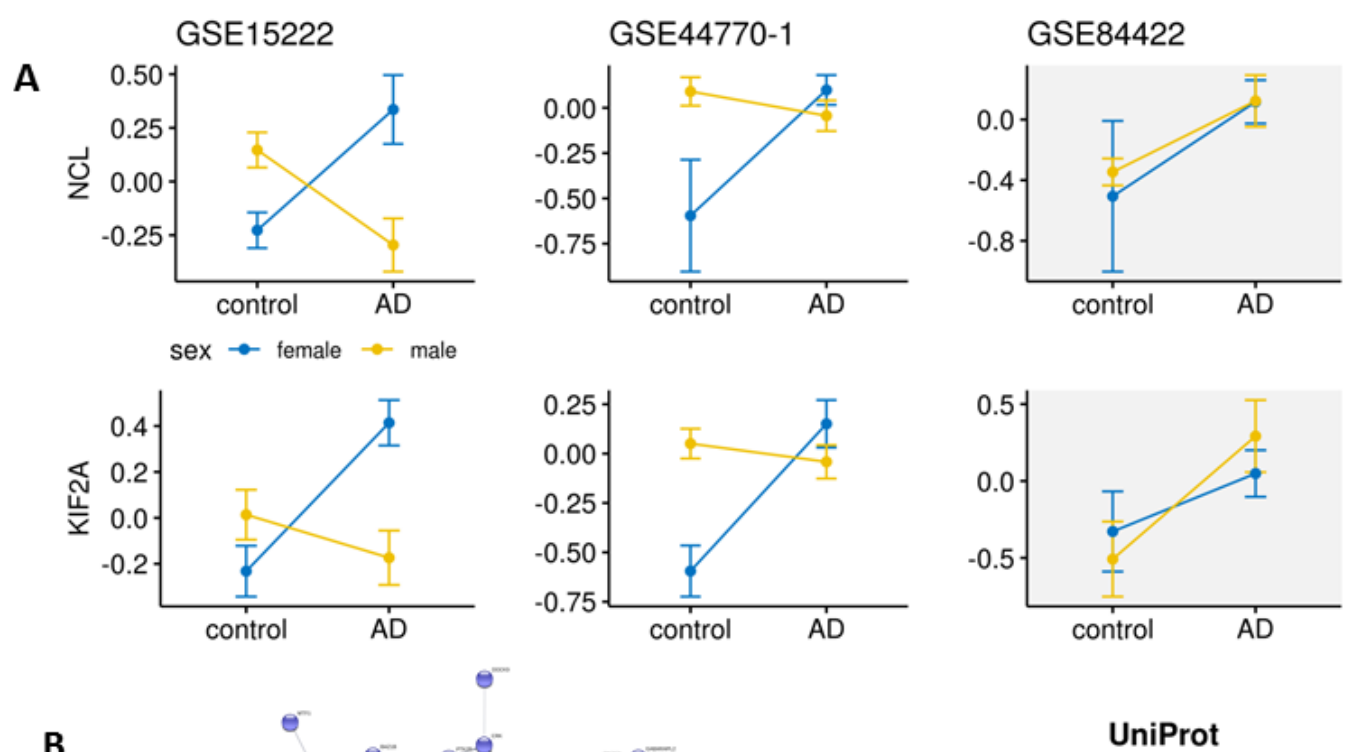

B
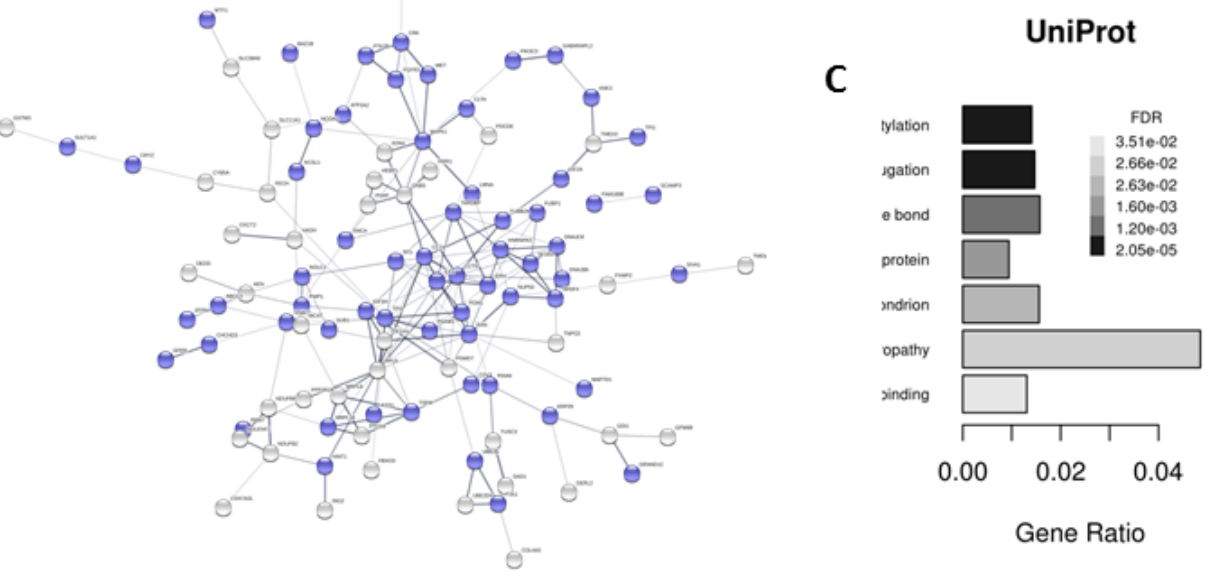

Figure 1. A) Gene expression residuals, after adjusting by sex, disease state, age and surrogate variables, as a function of disease state and stratified by sex. Sex-AD interactions for NCL and KIF2A expression are significant in the meta-analysis, corrected for multiple comparisons, and consistent between GSE15222 and GSE44770/ GSE44771, with nominally 
significant associations each. No significant associations were observed in GSE84422 (grey background). B) Protein-protein STRING network. The network was constructed for the genes with significant differential expression for the sex-AD interaction in post-mortem cortical tissue. The genes showed transcriptome-wide significance in the meta-analysis across three studies and were also significant in two studies. 68 connected genes are shown in blue, from a total of 76 significant genes within the protein phosphorylation pathway. C) Enrichment analyses for the UniProt database of biochemical pathways. Genes in phosphoprotein are colored in the network. 